\title{
The Relation between the Severity of Pain and Common Symptoms in Patients with Metastatic Cancer
}

\author{
Nehal G. El-Sayed ${ }^{1}$, Wael H. El-Sawy ${ }^{1}$, Ahmed E El-Azony ${ }^{1}$, Enas Noshy $^{2}$, Samy A. \\ Alsirafy ${ }^{2}$
}

1 Clinical Oncology Department, Faculty of Medicine, Zagazig University, Zagazig, Egypt; 2 Palliative Medicine Unit, Kasr Al-Ainy Center of Clinical Oncology \& Nuclear Medicine, Kasr AlAiny School of Medicine, Cairo University, Cairo, Egypt

Background: There is a relation between the severity of pain and common symptoms in patients with metastatic cancer. Aim: This study was done to explore this relation among Egyptian patients with advanced cancer.

Methods: The study included 120 adult metastatic cancer patients with pain from two cancer centers in Cairo and Sharkia, Egypt. Pain and other common symptoms were assessed using the Arabic version of the Edmonton Symptom Assessment System (ESAS). The Eastern Cooperative Oncology Group (ECOG) performance scale was used to assess performance status.

Results: The prevalence of ESAS symptoms was high among patients with cancer pain (tiredness, 94\%; drowsiness, 63\%; nausea, $60 \%$; lack of appetite, 77\%; shortness of breath, 53\%; depression, $88 \%$; anxiety, $83 \%$; poor wellbeing, $96 \%$ ). The ECOG performance scale was 1 in $21(17.5 \%)$ patients, 2 in $57(47.5 \%), 3$ in $38(31.7 \%)$ and 4 in $4(3.3 \%)$. The average ESAS score was $33.9 \pm 13.8,48.9 \pm 14.7,58 \pm 15.4$ and $70 \pm 5.5$ among patients with ECOG score 1, 2, 3 and 4; respectively $(p<0.001)$. There was no significant difference in the average score of any of the ESAS items according to the site of metastases. There was a significant positive correlation between the pain score and the scores of tiredness $(\mathrm{p}<0.001)$, nausea $(\mathrm{p}=0.037)$, lack of appetite $(\mathrm{p}<0.001)$, shortness of breath $(\mathrm{p}=0.001)$, depression $(\mathrm{p}<0.001)$, anxiety $(\mathrm{p}<0.001)$ and poor wellbeing $(\mathrm{p}<0.001)$.

Conclusion: Egyptian patients with cancer pain experience high symptom burden. The severity of pain strongly correlates with the presence and severity of other ESAS symptoms. Systematic assessment of other symptoms is indispensable in patients with cancer pain for proper control of symptoms and improving quality of life.

Keywords: Metastatic cancer, Pain, Symptoms, Performance status Corresponding author: Nehal G. El-Sayed, Clinical Oncology Department, Faculty of Medicine, Zagazig University, Zagazig, Egypt; nehaljamal80@yahoo.com

Submitted: 24-July-2017, Revised: 4-August-2017, Accepted: 5-August-2017, Published online: 13-August-17

\section{INTRODUCTION}

The World Health Organization defines palliative care as "an approach that improves the quality of life of patients and their families facing the problem associated with life-threatening illness, through the prevention and relief of suffering by means of early identification and impeccable assessment and treatment of pain and other problems, physical, psychosocial and spiritual" ${ }^{1}$.

Patients with advanced cancer usually suffer from multiple physical and psychological symptoms ${ }^{2}$. In a study from Egypt, >70\% of newly diagnosed incurable cancer patients reported more than 10 symptoms and the average number of symptoms per patient was 16.8 in the same study ${ }^{3}$. Cancer patients near end of life report more severe symptoms than other cancer patients ${ }^{4}$. Hui et al found that patients in the last week of life still suffer from high symptom burden mainly dyspnea, fatigue and drowsiness; and according to them, these symptoms may be unavoidable in this group of patients in spite of high quality supportive care ${ }^{5}$.

The prevalence of pain in cancer patients varies significantly between studies. In a systematic review on the prevalence of pain in cancer patients at different stages of their disease, pain prevalence ranged from 33$64 \%{ }^{6}$.The prevalence of pain varies between $50 \%$ at time of diagnosis to $75 \%$ in the advanced stages according to the International Association for the Study of Pain (IASP) files 2008-2009 7 .

Many assessment tools were developed to evaluate the symptom burden in advanced cancer patients including the Distress Thermometer (DT) ${ }^{8}$, the Memorial Symptom Assessment Scale (MSAS) ${ }^{9}$, the Edmonton Symptom Assessment System (ESAS) ${ }^{10}$, the European Organization for Research and Treatment of Cancer (EORTC) tools ${ }^{11}$, the Functional Assessment of Cancer Therapy (FACT) tools ${ }^{12}$, and McGill quality of life (QoL) questionnaire ${ }^{13}$. The ESAS is a simple tool to assess symptom burden. It was translated to many languages including Arabic and was validated in Egyptian patients where $65 \%$ of patients said the questionnaire was clear and $66 \%$ were able to answer it without help ${ }^{14}$.

Proper assessment of different symptoms and their relation to each other is important to control symptoms and to improve the QoL in palliative care patients ${ }^{15}$. For 
example, patients with depressive mood have higher symptom burden than other patients ${ }^{16}$.

The word symptom cluster is a relatively new concept and means that two or more symptoms are closely related and may have or not have a common cause. Once used in the clinical setting, it may have positive implications on diagnosis and treatment ${ }^{17}$. In the study done by Gift et al, the symptom cluster in a group of lung cancer patients was an independent predictor of patient death ${ }^{18}$. Chen et al identified 3 symptom clusters in cancer patients: sickness cluster (fatigue, sleep disturbance, lack of appetite and drowsiness), gastrointestinal symptom cluster (nausea and vomiting), and emotional symptom cluster (sadness and distress) ${ }^{19}$. The functional status of patients correlate negatively with sickness cluster, gastrointestinal symptoms are more common in patients under chemotherapy and patients with anxiety or depression had higher scores of emotional clusters. Different symptom clusters were described in other studies ${ }^{20,21}$.

In this study, we investigated the symptom burden in cancer patients with pain and the relation between pain and other symptoms in the ESAS.

\section{METHODS}

The study included adult patients with solid tumors and evidence of distant metastases. All included patients had pain and gave a verbal informed consent to participate in the study. Patients were recruited from two cancer centers in Cairo (Kasr Al-Ainy Center of Clinical Oncology and Nuclear Medicine [NEMROCK], Kasr Al-Ainy School of Medicine, Cairo University) and Sharkia (Department of Clinical Oncology, Faculty of Medicine, Zagazig University, Sharkia) governorates. The study was approved by the ethics committees in the two institutions where it took place.

A data collection sheet was constructed including age, sex, primary cancer, site of metastases and performance status which was assessed using the Eastern Cooperative Oncology Group (ECOG) performance scale ${ }^{22}$. The validated Arabic version of the ESAS was used to asses the severity of pain and other common symptoms including: tiredness, nausea, depression, anxiety, drowsiness, appetite, poor wellbeing and shortness of breath. The severity of each ESAS item is rated from 0 to 10 on a numerical scale, 0 meaning that the symptom is absent and 10 that it is of the worst possible severity. Due to the relatively high illiteracy rate, patients were interviewed by investigators $\mathrm{NE}$ and EN to complete the data collection sheets.

Data was expressed as percentage, mean \pm standard deviation or median and range where appropriate. Independent sample t-test was used to test the significance of difference in the mean ESAS score between two groups and one-way ANOVA between $>2$ groups. Pearson's correlation was used to test the relation between the severity of pain and that of other ESAS symptoms. Linear regression analysis was performed to identify symptoms that correlate with pain severity independently. A P value <.05 was considered significant. Statistical analysis was performed using SPSS, version 14.0. (SPSS Inc, Chicago, Illinois).

\section{RESULTS}

The study included 120 adult patients with metastatic cancer who were recruited from October 2016 to April 2017. The characteristics of patients are shown in Table 1.

Table 1: Patients' characteristics

\begin{tabular}{clll}
\hline & & No. & \% \\
\hline Age & & \\
\hline Sex & Median (range) & $57(25-92)$ & \\
\hline & Male & 59 & 49.2 \\
\cline { 2 - 3 } & Female & 61 & 50.8 \\
\hline Primary cancer & & \\
\hline Breast & 29 & 24.2 \\
\hline Liver & 19 & 15.8 \\
\hline Lung & 15 & 12.5 \\
\hline Colorectal & 11 & 9.2 \\
\hline Pancreas & 8 & 6.7 \\
\hline Ovary & 7 & 5.8 \\
\hline Urinary bladder & 6 & 5 \\
\hline Other & 25 & 20.8 \\
\hline Site of metastases & & \\
\hline Bone & 73 & 60.8 \\
\hline Liver & 40 & 33.3 \\
\hline Lung & 21 & 17.5 \\
\hline Brain & 11 & 9.2 \\
\hline Lymph nodes & 5 & 4.2 \\
\hline Other & 9 & 7.5 \\
\hline
\end{tabular}

All included patients had cancer pain. The average severity of pain and other symptoms assessed by the ESAS are illustrated in Table 2.

The ECOG performance scale was 1 in $21(17.5 \%)$ patients, 2 in $57(47.5 \%), 3$ in $38(31.7 \%)$ and 4 in 4 (3.3\%). The total ESAS score differed significantly according to the ECOG performance scale (Figure 1). The average ESAS score was $33.9 \pm 13.8,48.9 \pm 14.7$, $58 \pm 15.4$ and $70 \pm 5.5$ among patients with ECOG 1, 2, 3 and 4 ; respectively $(\mathrm{p}<0.001)$.

Table 2: Prevalence and severity of symptoms assessed by the ESAS.

\begin{tabular}{lll}
\hline Symptom & Severity & Prevalence \\
\cline { 2 - 3 } & Mean $( \pm$ SD) & No. $(\%)$ \\
\hline Pain & $7.82( \pm 1.7)$ & $120(100)$ \\
\hline Tiredness & $7.27( \pm 2.57)$ & $113(94.2)$ \\
\hline Drowsiness & $3.88( \pm 5.56)$ & $75(62.5)$ \\
\hline Nausea & $3.33( \pm 3.33)$ & $72(60)$ \\
\hline Lack of appetite & $5.53( \pm 3.56)$ & $92(76.7)$ \\
\hline Shortness of breath & $3.36( \pm 3.65)$ & $63(52.5)$ \\
\hline Depression & $5.93( \pm 3.19)$ & $105(87.5)$ \\
\hline Anxiety & $5.63( \pm 3.4)$ & $100(83.3)$ \\
\hline Poor wellbeing & $7.13( \pm 2.49)$ & $115(95.8)$ \\
\hline Total ESAS score & $49.86( \pm 16.95)$ & \\
\hline
\end{tabular}

ESAS: Edmonton Symptom Assessment System 
There was no significant difference in the average score of any of the ESAS items according to presence of lymph nodes, liver or bone metastases.

Drowsiness was significantly higher $(\mathrm{p}=0.007)$ in the presence of brain metastases and shortness of breath was significantly higher $(\mathrm{p}=0.032)$ in the presence of lung metastases.

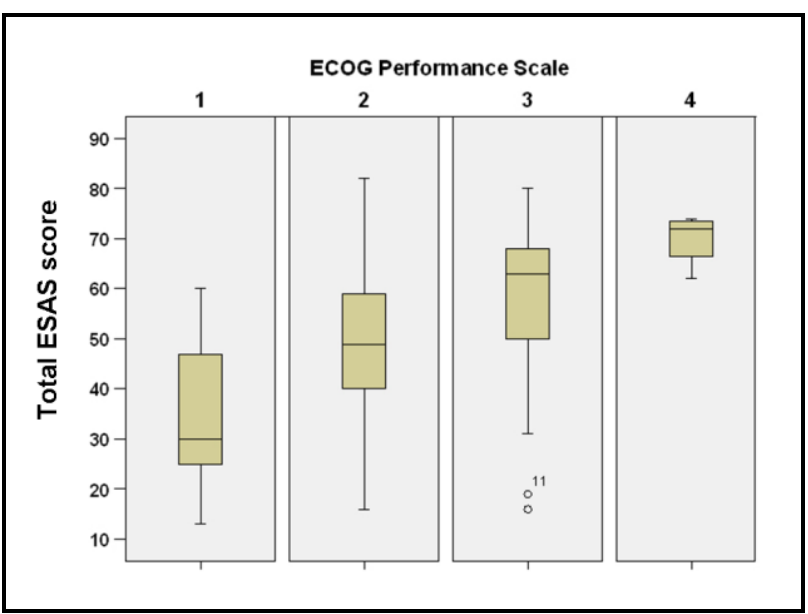

Figure 1. Box plot representation of the relation between ESAS (Edmonton Symptom Assessment System) and ECOG (Eastern Cooperative Oncology Group) performance scale

In univariate analysis, there was a significant positive association between the pain score and the scores of all other ESAS items except drowsiness (Table 3). In multivariate analysis, the only item that was associated significantly with pain was the "poor wellbeing" item $(\mathrm{p}=0.018)$.

Table 3. Correlation between pain score and other ESAS items scores.

\begin{tabular}{lll}
\hline Other ESAS items & \multicolumn{2}{l}{ Pain } \\
\cline { 2 - 3 } & $\begin{array}{l}\text { Pearson's } \\
\text { correlation }\end{array}$ & p value \\
\hline Tiredness & 0.393 & $<0.001$ \\
\hline Drowsiness & 0.115 & 0.212 \\
\hline Nausea & 0.191 & 0.037 \\
\hline Lack of appetite & 0.391 & $<0.001$ \\
\hline Shortness of breath & 0.309 & 0.001 \\
\hline Depression & 0.468 & $<0.001$ \\
\hline Anxiety & 0.453 & $<0.001$ \\
\hline Poor wellbeing & 0.479 & $<0.001$ \\
\hline
\end{tabular}

ESAS: Edmonton Symptom Assessment System

\section{DISCUSSION}

The current study showed that Egyptian metastatic cancer patients with pain experience high physical and psychological symptom burden. Most of the included patients suffered from tiredness (94\%), anxiety $(87.5 \%)$, and depression $(83 \%)$. These results are much higher than those reported by Barbara et al ${ }^{4}$ (tiredness $24 \%$, $43 \%$ anxiety $43 \%$ and depression $55 \%$ ) and Alsirafy et al ${ }^{3}$ (tiredness $61 \%$, anxiety $61 \%$, depressed $\operatorname{mood} 57 \%$ ). This might be related to the fact that all included patients suffered from pain. Also, both studies included patients earlier in the course of their disease.

In this study, there was a correlation between the severity of pain and that of tiredness, lack of appetite, depression, anxiety and poor wellbeing. This was previously reported by Tavoli et al, where higher score of cancer pain was associated with higher depression, and lower global QoL ${ }^{23}$. In an Italian study, there was a linear decrease in the mean global QoL from 64.9 in patients without pain to 36.4 in patients with severe pain $(\mathrm{p}<0.001)^{24}$. The relation between pain, anxiety, and depression is complex and needs attention due to its implication on the proper assessment of patients and hence on the outcome of treatment of these symptoms. Pain increases the feeling of depression and anxiety ${ }^{24}$. At the same time, patients with depression and anxiety experience more severe pain ${ }^{25}$.

Lack of appetite occurred in $76 \%$ of our patients with an average ESAS score of 5. This is not far from that reported by Karabulut et al ${ }^{26}$, where $88 \%$ reported loss of appetite and Nayak et al who described loss of appetite in $89 \%$ of patients ${ }^{27}$. On the other hand, it is higher than that reported in a review by Teunissen et al where lack of appetite occurred in $53 \%$ of patients ${ }^{28}$. The lower rate reported by Teunissen et al may be related to the diversity of studies and different assessment methods in the studies included in their systematic review.

In our study, the total ESAS correlated significantly with the performance status. This is in accordance with a Japanese study where ESAS-r was significantly higher in patients with an ECOG performance status of 2-4 than in those with a performance status of 0 and $1^{29}$. Similarly, in the study done by Shalini et al, patients with lower performance status measured by both ECOG and Karnofsky performance scales had worse QoL scores in symptomatic pancreatic pain ${ }^{30}$. This can be explained by the fact that patients at the end of life are very sick and have high symptom burden which may contribute to the decline in performance status and QoL scores. However in an Italian study, there was a trend for the ESAS score to be higher in poor performance status but did not reach statistical significance ${ }^{31}$.

Our study has many limitations. First, the ESAS is a short instrument assessing only nine symptoms. Further studies are required with more comprehensive tools to properly assess the relation between different symptoms in cancer patients. Another limitation is the inclusion of only patients suffering from pain, which make our results different form other studies. The recruitment of patients was done by convenience method. More systematic recruitment methods can be used in future studies to avoid selection bias. Also, patients were interviewed only once and no follow up interviews were done after pain treatment to assess the difference in symptom burden and performance status after pain control. This may result in better understanding of the relation between pain and other symptoms. 


\section{Conclusion}

Cancer pain in Egyptian patients with metastatic cancer is associated with high physical and psychological symptom burden. The severity of pain correlates with the severity of other symptoms present in the ESAS. Proper assessment of symptoms, physical and psychological, is indispensable for the proper control of cancer pain and other symptoms and improving QoL of advanced cancer patients.

\section{REFERENCES}

1. World Health Organization. WHO Definition of Palliative Care. Available from http://www.who.int/cancer/palliative/definition/en/.

Accessed: 18-July-2017.

2. Schmidt H, Merkel D, Koehler M, et al. PRO-ONKOselection of patient-reported outcome assessments for the clinical use in cancer patients-a mixed-method multicenter cross-sectional exploratory study. Support Care Cancer. 2016; 24(6): 2503-2512.

3. Alsirafy SA, Abd El-Aal HH, Farag DE, Radwan RH, ElSherief WA, Fawzy R. High symptom burden among patients with newly diagnosed incurable cancer in a developing country. J Pain Symptom Manage. 2016; 51 (5): e1-5.

4. Barbera L, Seow H, Howell D, et al. Symptom burden and performance status in a population-based cohort of ambulatory cancer patients .Cancer. 2010: 116(24): 57675776.

5. Hui D, dos Santos R, Chisholm GB, Bruera E. Symptom expression in the last seven days of life among cancer patients admitted to acute palliative care units. J Pain Symptom Manage. 2015; 50(4): 488-494.

6. van den Beuken-van Everdingen $\mathrm{MH}$, de Rijke JM, Kessels AG, Schouten HC, van Kleef M, Patijn J. Prevalence of pain in patients with cancer: a systematic review of the past 40 years. Ann Oncol. 2007; 18 (9): 1437-1449.

7. Global Year against Cancer Pain. Epidemiology of cancer pain. Available from https://www.iasp.pain.org/files/Content/ContentFolders/G lobalYearAgainstPain2/CancerPainFactSheets/Epidemiol ogy_Final.pdf. Accessed: 20-July-2017.

8. Roth AJ, Kornblith AB, Batel-Copel L, Peabody E, Scher HI, Holland JC. Rapid screening for psychologic distress in men with prostate carcinoma: a pilot study. Cancer. 1998; 82(10): 1904-1908.

9. Portenoy R, Thaler H, Kornblith A, et al. The Memorial Symptom Assessment Scale: an instrument for the evaluation of symptom prevalence, characteristics and distress. Eur J Cancer. 1994; 30A(9): 1326-1336.

10. Bruera E, Kuehn N, Miller M, Selmser P, Macmillan K. The Edmonton Symptom Assessment System (ESAS): a simple method for the assessment of palliative care patients. J Palliat Care. 1991; 7(2): 6-9.

11. Aaronson N, Ahmedzai S, Bergman B, et al. The European Organization for Research and Treatment of Cancer QLQ-C30: a quality-of-life instrument for use in international clinical trials in oncology. J Natl Cancer Inst. 1993; 85(5): 365-376.

12. Cella D, Tulsky D, Gray G, et al. The Functional Assessment of Cancer Therapy scale: development and validation of the general measure. J Clin Oncol. 1993; 11(3): 570-579.

13. Cohen SR, Mount BM, Strobel MG, Bui F. The McGill Quality of Life Questionnaire: a measure of quality of life appropriate for people with advanced disease. A preliminary study of validity and acceptability. Palliat Med. 1995; 9(3): 207-219.

14. Salem DA, Adel AM, Essa AE, Alorabi MO, Elsayed ZM Feasibility and reliability of the revised Edmonton Symptom Assessment System (ESAS-r) in Egyptian patients with advanced cancer: A single institutional experience. Oncol Transl Med. 2016; 2(3): 132-137.

15. Fleishman SB. Treatment of Symptom Clusters: Pain, Depression, and Fatigue. J Natl Cancer Inst Monogr.2004; (32): 119-123.

16. Delgado-Guay M, Parsons HA, Li Z, Palmer JL, Bruera E. Symptom distress in advanced cancer patients with anxiety and depression in the palliative care setting. Support Care Cancer. 2009; 17(5): 573-579.

17. Fan G. Filipzak L, Chow E. Symptom clusters in cancer patients: a review of literature. Curr Oncol. 2007; 14(5): 173-179

18. Gift AG, Stommel M, Jablonski A, Given W. A cluster of symptoms over time in patients with lung cancer. Nurs Res. 2003; 52(6): 393-400.

19. Chen ML, Tseng HC. Symptom clusters in cancer patients Support Care Cancer. 2006; 14(8): 825-830.

20. Wang XS, Wang Y, Guo H, Mendoza TR, Hao XS, Cleeland CS. Chinese version of the M. D. Anderson Inventory: validation and application of symptom measurement in cancer patients. Cancer. 2004; 101(8): 1890-901.

21. Cleeland CS, Mendoza TR, Wang XS, et al. Assessing symptom distress in cancer patients: the MD Anderson symptom inventory. Cancer. 2000; 89(7): 1634-1646.

22. Oken MM, Creech RH, Tormey DC, et al. Toxicity and response criteria of the Eastern Cooperative Oncology Group. Am J Clin Oncol. 1982; 5(6): 649-656.

23. Tavoli A, Montazeri A, Roshan R, Tavoli Z, Melyani M. Depression and quality of life in cancer patients with and without pain: the role of pain beliefs. BMC Cancer. 2008; 8:177.

24. Di Maio M, Gridelli C, Gallo C, et al. Prevalence and management of pain in Italian patients with advanced non-small-cell lung cancer. Br J Cancer. 2004; 90(12): 2288-2296.

25. Sist TC, Florio GA, Miner MF, Lema MJ, Zevon MA. The relationship between depression and pain language in cancer and chronic non-cancer pain patient. J Pain Symptom Manage.1998; 15(6): 350-358.

26. Karabulut N, Erci B, Ozer N, Ozdemir S. Symptom clusters and experiences of patients with cancer. J Adv Nurs. 2010; 66(5): 1011-1021.

27. Nayak MG, George A, Vidyasagar MS, et al. Symptoms experienced by cancer patients and barriers to symptom management. Indian J Palliat Care. 2015; 21(3): 349-354.

28. Teunissen SC, Wesker W, Kruitwagen C, de Haes HC, Voest EE, de Graeff A. Symptom Prevalence in Patients with Incurable Cancer: A Systematic Review. J Pain Symptom Manage.2007; 34(1): 94-104.

29. Yokomichi N, Morita T, Nitto A, et al. Validation of the Japanese version of the Edmonton Symptom Assessment System-Revised. J Pain Symptom Manage. 2015; 50(5): 718-723.

30. Moningi S, Walker AJ, Hsu CC, et al. Correlation of clinical stage and performance status with quality of life in patients seen in a pancreas multidisciplinary clinic. $\mathrm{J}$ Oncol Pract. 2015; 11(2): e216-e221.

31. Moro C, Brunelli C, Miccinesi G, et al. Edmonton symptom assessment scale: Italian validation in two palliative care settings. Support Care Cancer. 2006; 14(1): 30-37. 